\title{
Trombosis venosa cortical aislada. Comunicación de dos pacientes
}

\author{
Héctor Miranda $V^{1}$, Patricio Mellado $\mathrm{T}^{1}$, \\ Patricio Sandoval $\mathbf{R}^{\mathbf{1}}$, Isidro Huete $\mathbf{L}^{2}$. \\ Isolated cortical vein thrombosis. \\ Report of two cases
}

Isolated cortical vein thrombosis is an uncommon presentation of central venous thrombosis. We report two females, aged 29 and 40 years, with isolated cortical vein thrombosis. Both presented with a focal neurological deficit and focal seizures that became generalized. The diagnosis was made with magnetic resonance imaging. Both had a history of oral contraceptive use. Both had a rapid response to unfractionated heparin. One patient had an antiphospholipid syndrome as a possible etiology. The most common manifestations of this disease are a transient or recurrent neurological deficit, visual disturbances and focal or generalized seizures, usually without intracanial hypertension. Neuroimages show ischemic abnormalities that do not follow an arterial vascular territory, often with an early hemorrhagic component. There is a good clinical response to heparin (Rev Méd Chile 2007; 135: 1313-17).

(Key words: Antiphospholipid syndrome; Intracranial thrombosis; Venous thrombosis)

Recibido el 23 de junio, 2006. Aceptado el 21 de noviembre 2006.

${ }^{1}$ Departamento de Neurología, Intermedio Neurológico y ${ }^{2}$ Departamento de Radiología, Hospital Clínico Pontificia Universidad Católica de Chile. Santiago de Chile.

$\mathrm{L}^{2}$ a trombosis venosa cortical aislada (TVCA) es una de las formas más infrecuentes de la trombosis venosa cerebral (TVC), con sólo comunicaciones anecdóticas en la literatura. Su diagnóstico es difícil, clínicamente se caracteriza por un déficit neurológico focal, acompañado de crisis convulsivas también focales que pueden generalizarse o un estado epiléptico parcial simple, frecuentemente de tipo motor. Las alteraciones que se observan en la resonancia magnética de encéfalo son características y la respuesta a la terapia anticoagulante es habitualmente favorable. Descri-

Correspondencia a: Héctor Miranda Vera. San Isidro 379, Depto. 24. Santiago. Fono: 02-4411565 - 09-2192802. Fax: 6326221. E mail: hdmirand@puc.cl bimos dos pacientes con TVCA confirmada por neuroimágenes y comparamos sus hallazgos con los descritos en la literatura.

\section{CASOS CLÍNICOS}

Paciente 1. Mujer de 29 años, diestra, con antecedentes de tabaquismo y usuaria de anticonceptivos orales (ACO) desde hacía 4 meses. Consultó por disminución de fuerzas y de sensibilidad en la extremidad superior derecha, a la que se agregó movimientos involuntarios del mismo hemicuerpo y que progresó a una crisis convulsiva tónicoclónica generalizada de un minuto de duración. El examen general de ingreso era normal, en la evaluación neurológica se encontraba vigil, orientada, sin alteraciones de lenguaje, con leve hemi- 
paresia faciobraquiocrural, hemihipoestesia con agrafoestesia y signo de Babinski, derechos. El estudio de laboratorio general de ingreso fue normal. Se realizó una resonancia magnética (RM) de encéfalo que mostró en secuencias T2 y FLAIR una hiperintensidad cortical frontal izquierda, en T1 con gadolineo no hubo un refuerzo significativo, en T2 gradiente una hipointensidad lineal cortical sugerente de una vena trombosada y en la fase venosa de la angio-RM, un defecto de llene de una vena cortical en la convexidad izquierda (Figura 1).

Se planteó el diagnóstico de TVCA y se inició terapia anticoagulante con heparina no fraccionada intravenosa (iv) y anticonvulsivante con fenitoína oral. El estudio etiológico sólo mostró anticuerpos anticardiolipinas con valores de IgG $1,1 \mathrm{gpl}$ (normal 0-15) e IgM 27,7 mpl (normal 015), planteándose el diagnóstico de un probable síndrome antifosfolípido primario. Al tercer día de tratamiento la paciente se encontraba sin déficit neurológico, fue dada de alta al quinto día, asintomática, con fenitoína y tratamiento anticoagulante oral.
Paciente 2. Mujer de 40 años, usuaria de ACO y antecedentes de linfoma de Hodgkin tipo esclerosis nodular 1-A 30 años antes, tratada con quimioterapia y radioterapia y carcinoma submandibular indiferenciado 12 años antes tratado con cirugía y radioterapia local. La paciente se encontraba curada de ambas patologías. Consultó en un centro hospitalario por estado epiléptico parcial complejo, con clonías de hemicuerpo derecho de $24 \mathrm{~h}$ de evolución. Se realizó una tomografía computada (TC) de encéfalo, que fue informada como hemorragia subaracnoídea en los surcos de la convexidad frontal precentral izquierda. Se inició terapia anticonvulsivante con fenitoína y se debió agregar ácido valproico por persistencia de clonías del hemicuerpo derecho; evolucionó con hemiparesia braquiocrural derecha. La RM cerebral con difusión y T2 mostró una hiperintensidad en la región precentral y postcentral izquierda, compatibles con infartos corticales, la angiorresonancia mostró indemnidad las arterias extra e intracraneanas y de los senos venosos. Fue trasladada a nuestro centro $48 \mathrm{~h}$ después de iniciado el cuadro, a su ingreso destacaba: disartria, afasia

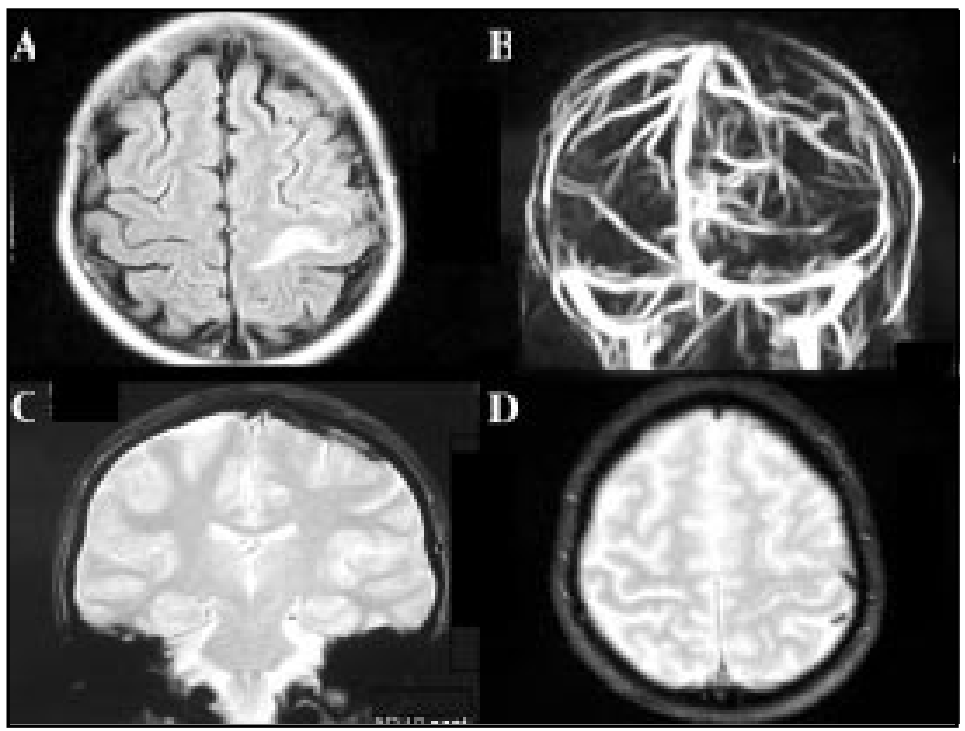

Figura 1. A: Secuencia FLAIR de resonancia magnética: se observa una hiperintensidad en la corteza y surco prerrolándico izquierdo. B: Angiorresonancia en fase venosa: Muestra una vena cortical izquierda con un defecto de llene. C: T2 gradiente coronal. D: T2 gradiente axial: En ambos se observa una imagen tubular cortical izquierda hipointensa sugerente de trombosis de una vena cortical. 
expresiva, hemiparesia fláccida faciobraquiocrural y signo de Babinski derechos. El estudio de laboratorio general de ingreso fue normal. El electroencefalograma mostró una lentitud izquierda intermitente máxima anterior. Al reevaluar la RM previamente realizada se observó una imagen sugerente de una trombosis de venas corticales aisladas frontales izquierdas (Figura 2). Se decidió realizar una angiografía por sustracción digital, la que confirmó la exclusión de venas corticales frontales izquierdas, con indemnidad de seno sagital superior (Figura 2). Se decidió iniciar anticoagulación con heparina intravenosa no fraccionada. El estudio etiológico completo, incluyendo trombofilia, fue normal. Al décimo día de anticoagulación, la paciente presentaba sólo una leve hemiparesia braquiocrural derecha, se traslapó a anticoagulación oral y fue dada de alta.

\section{DisCUSIÓN}

La TVC es una patología infrecuente y de difícil diagnóstico, las primeras descripciones fueron realizadas por Ribes en 1825 y Abercrombie en $1828^{1,2}$. Actualmente se estima que tres o cuatro casos nuevos de TVC se atienden cada año en un servicio de neurología 1,3,4. En nuestra unidad de neurointermedio se han presentado 3 casos anualmente entre 2001 y 20045. La distribución por sexos muestra una relación entre mujeres y hombres de 1,2-1,6:1, con leve aumento entre los 20 y 35 años en las mujeres ${ }^{2}$. En nuestra serie recientemente publicada, los nueve pacientes eran mujeres, con una edad promedio de 40,6 414,6 años ${ }^{5}$.

Las venas más frecuentemente comprometidas son: seno sagital superior (SSS) y seno lateral (SL), seguidos por las venas del sistema galénico, seno cavernoso y petrosos, y menos frecuentemente por el compromiso aislado de venas corticales y venas cerebelosas ${ }^{2}$.

En 1880, Raymond realizó la primera descripción detallada de la TVCA ${ }^{4}$. En la actualidad los reportes de TVCA son escasos, su incidencia en series de TVC es de $2 \%{ }^{3,6-8}$. Las consecuencias de la TVCA son variables, puede ser asintomática y sin lesiones pesquisables debido al desarrollo de circulación colateral, presentar sólo un edema

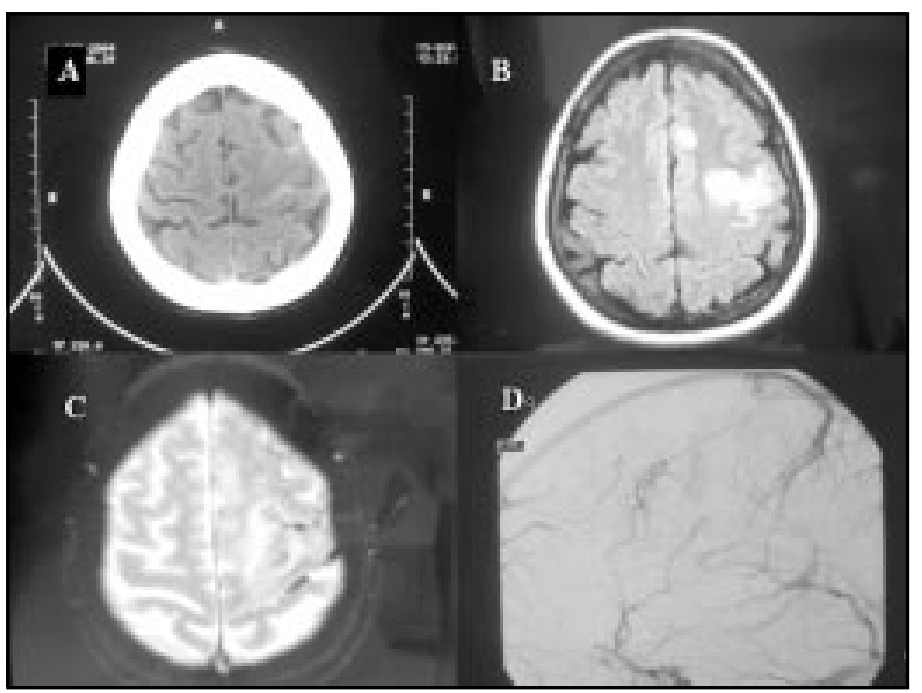

Figura 2. A: Tomografía computada de encéfalo que muestra una hipodensidad subcortical frontal izquierda y una hiperdensidad de surcos frontales izquierdos. B: Secuencia FLAIR: se observa hiperintensidad en la corteza y surcos prerrolándicos izquierdos. C: T2 gradiente axial. Se observa imagen tubular cortical izquierda hipointensa sugerente de trombosis de una vena cortical. D: Angiografía por sustracción digital en fase venosa de hemisferio izquierdo. Se observa una brusca detención del contraste en una vena cortical. 
localizado asintomático e incluso en pacientes con un infarto venoso establecido por imágenes, tener una recuperación completa ${ }^{3,4}$.

El número y localización de las venas corticales no son constantes y los infartos venosos corticales pueden afectar cualquier región del encéfalo ${ }^{3,6}$. Las localizaciones más frecuentes son las regiones: rolándica, parieto-occipital y temporal posterior, adyacentes al SSS y SL ${ }^{3}$. En el caso de nuestras pacientes, ambas tuvieron un compromiso de la región paracentral rolándica izquierda (Figuras 1 y 2).

Las manifestaciones clínicas de la TVCA dependen de la región cortical comprometida, es frecuente que el déficit neurológico focal se asocie a crisis convulsivas focales, las que pueden generalizarse e incluso evolucionar a un estado epiléptico, el que habitualmente es de tipo parcial, pudiendo ser parcial complejo o generalizado ${ }^{6,9-12}$. En nuestras pacientes, se observaron signos focales característicos de la región prerrolándica izquierda asociados a crisis focales, en el primer caso con una generalización secundaria y en la segunda paciente con evolución a un estado epiléptico parcial complejo refractario a fenitoína.

Existen más de 100 factores etiopatogénicos asociados a la TVC ${ }^{3}$. Destacan los ACO, los que se encuentran como único factor de riesgo de TVC en $10 \%$ de los casos y en $15 \%$ adicional asociado a otro factor de riesgo ${ }^{2}$. Nuestras dos pacientes eran usuarias de ACO, la primera tenía además un probable síndrome antifosfolípido. La segunda paciente tenía como antecedentes un linfoma y un carcinoma submandibular, los que también se asocian a TVC, sin embargo, debido a largo tiempo libre de enfermedad, 30 y 12 años, respectivamente, es improbable que ellos estuvieran asociados a la TVCA. Por último, esta misma paciente fue sometida en dos oportunidades a radioterapia la que no incluyó el cráneo. Para el carcinoma submandibular se irradió la región cervical, lo que puede asociarse a arteriopatía actínica local, sin embargo, la angiomesonancia y la angiografía por sustracción digital mostraron arterias y venas cervicales de aspecto normal.

La TC de encéfalo es habitualmente normal en los casos de TVCA. Sin embargo, se pueden observar algunos signos inespecíficos como áreas hipodensas córtico-subcorticales sugerentes de un infarto o áreas hiperdensas en los surcos que pueden confundirse con una hemorragia subaracnoidea de la convexidad y que comesponden a venas con estasis y dilatadas.
Estas últimas características se pudieron observar en nuestra segunda paciente. Otros signo en la TC sugerente de una TVCA es el denominado signo de la cuerda, donde se observa una hiperdensidad espontánea de tipo lineal en la convexidad del hemisferio y que se debe a un trombo en una vena cortical ${ }^{2}$.

La RM de encéfalo es considerada el examen gold standard para el diagnóstico de la TVC, con una sensibilidad y especificidad mayor a 95\%2,3,13. En los casos de TVCA, se puede observar el infarto, el edema secundario y la transformación hemorrágica debido a la alta sensibilidad que tiene T2 gradiente. Por último, como en nuestras dos pacientes, la secuencia T2 gradiente puede mostrar la trombosis de la vena cortical, al observarse una estructura tubular hipointensa en la corteza. La angiorresonancia es útil para determinar el compromiso de senos venosos, lo que en nuestras pacientes fue negativo.

La angiografía por sustracción digital es usada cuando la RM no está disponible, está contraindicada o existe duda de su resultado, sin embargo, su interpretación es difícil en TVCA debido a que el número y localización de las venas corticales son inconstantes. Un signo útil para el diagnóstico de TVCA es la intermupción abrupta de una vena cortical como en el caso de nuestra segunda paciente ${ }^{2,4}$.

La anticoagulación con heparina es recomendada como tratamiento de la TVC por la mayoría de autores, incluso en casos con infarto venoso hemorrágico 1,2,14-17. La trombólisis local se recomienda como una terapia de rescate en casos de TVC extensas, con compromiso de senos venosos y refractarios al uso de heparina intravenosa no fraccionada $1,2,16,18$. En los casos reportados en la literatura de TVCA, al igual que nuestras dos pacientes, hubo una rápida respuesta a heparina intravenosa no fraccionada.

En síntesis, la TVCA es una entidad infrecuente y de difícil diagnóstico, que requiere de un alto grado de sospecha diagnóstica. Debe plantearse en pacientes con déficit neurológico focal asociado a crisis convulsivas focales o generalizadas y especialmente si se presenta con un estado epiléptico parcial. La $\mathrm{RM}$ de encéfalo es el examen de elección y debe buscarse una vena cortical distendida en T2 gradiente. Deben buscarse dirigidamente los factores asociados a TVC e iniciar precozmente una terapia anticoagulante con heparina intravenosa no fraccionada. El pronóstico es habitualmente bueno. 


\section{REFERENCIAS}

1. Álvarez-Pérez FJ, García-Sancho G, ZunzuneguiCostas J, López Facal M, Marín-Sánchez M, Rey Del CORRAL P. Trombosis venosa intracraneal: estudio de 16 casos y revisión de la bibliografía. Rev Neurol 2001; 33: 505-10.

2. MeLado P, Ñancupil $C$, Tapia J. Trombosis Venosa Encefálica. En: Castillo L, Romero C, Mellado P (eds). Cuidados Intensivos Neurológicos. Santiago: Editorial Mediterráneo, 2004; 352-64.

3. Bousser MG, RusSell R. Cerebral Venous Thrombosis. London: Saunders, 1997.

4. Bousser MG, BarnetT HJM. Cerebral venous thrombosis. En: Mohr JP, Weir B, Choi DW, Wolf PA, Grotta JC. (eds). Stroke: Pathophysiology, Diagnosis and Management. Philadelphia. Churchill Livingstone. 2004; 301-25.

5. Melado P, Court J, Godoy J, Mery V, Barnett C, ANDRESEN M ET AL. Características de la enfermedad cerebrovascular en un Servicio de Neurointermedio, en Chile. Análisis de 459 pacientes consecutivos. Rev Méd Chile 2005; 133: 1274-84.

6. Jacobs K, Moulin T, Bogousslavsky J, Woimant F, Dehaene I, Tatu L et al. The stroke syndrome of cortical vein thrombosis. Neurology 1996; 47: 376-82.

7. AMERI A, Bousser MG. Cerebral Venous Thrombosis. Neurol Clin 1992; 10: 87-111.

8. Cantú C, Barinagarrementería F. Cerebral venous thrombosis associated with pregnancy and puerperium; review of 67 cases. Stroke 1993; 24: 1880-4.

9. Vuimer F, Moulin T, Tatu L, Rumbach L, Bertrand MA. Isolated cortical vein thrombosis and acti- vated protein C resistance. Stroke 1996; 27: 1440-1.

10. Minadeo JP, Karaman BA. Headache: cortical vein thrombosis and response to anticoagulation. J Emerg Med 1999; 17: 449-53.

11. Tang O, Ng E, Wai Cheng P, Chung Ho P. Cortical vein thrombosis misinterpreted as intracranial haemorrhage in severe ovarian hyperstimulation syndrome. Hum Reprod 2000; 15: 1913-16.

12. Lu XY, Gabig TG, Bang NU. Combined heterozygosity of factor V Leiden and the G20210A prothrombin gene mutation in a patient with cerebral cortical vein thrombosis. Am J Hematol 2000; 64: 226-8.

13. Favrole P, Guichard JP, Crassard I, Bousser MG, СнавRiaT $\mathrm{H}$. Diffusion-weighted imaging of intravascular clots in cerebral venous thrombosis. Stroke 2004; 35: 99-103.

14. Stam J, De Brujn S, De Veber G. Anticoagulation for cerebral sinus thrombosis. Stroke 2003; 34: 1054-5.

15. Benamer H, Bone I. Cerebral venous thrombosis: anticoagulants or thrombolytic therapy. J Neurol Neurosurg Psychiatry 2000; 69: 427-30.

16. BOusSER MG. Cerebral venous thrombosis: nothing, heparin or local thrombolysis? Stroke 1999; 30: 481-3.

17. Einhaupl KM, Viuringer A, Meister W, Mehraein S, GarNer C, PeIJKofer M et al. Heparin treatment in sinus venous thrombosis. Lancet 1991; 338: 597-600.

18. Ciccone A, Canhao P, Falcao F, Ferro JM, Sterzi R. Thrombolysis for cerebral vein and dural sinus thrombosis. (Cochrane Review). In: The Cochrane Library, Issue 3, 2004. Chichester, UK: John Wiley \& Sons, Ltd. 